\title{
Transient abnormal myelopoiesis accompanied by hepatic fibrosis in two infants with Down syndrome
}

\author{
N Yagihashi, K Watanabe, S Yagihashi
}

\begin{abstract}
Two necropsy cases of Down syndrome are reported. These showed transient abnormal myelopoiesis accompanying characteristic hepatic sinusoidal fibrosis. Numerous megakaryocytes were found in the liver of one case, but not in the other. Only eight cases of Down syndrome with simultaneous occurrence of hepatic fibrosis and transient abnormal myelopoiesis have been reported. The cases described here showed slight fibrotic changes in the hyperplastic bone marrow, which were not found in the previously reported cases of transient abnormal myelopoiesis. (f Clin Pathol 1995;48:973-975)
\end{abstract}

Keywords: Transient abnormal myelopoiesis, Down syndrome, hepatic fibrosis, myelofibrosis.

Infants with Down syndrome often have acute but spontaneously regressing leukaemoid haematological changes, referred to as transient abnormal myelopoiesis, transient myeloproliferative disorder, or congenital leukaemoid reaction. A serious but very rarely reported ${ }^{1-4}$ complication is hepatic fibrosis. ${ }^{1}$ In previous necropsy studies, an increase in reticulin fibres in the bone marrow has not been described. We recently encountered two infants with Down syndrome and transient abnormal myelopoiesis accompanied by hepatic fibrosis. In addition there was a mild but distinct increase in reticulin in the hyperplastic bone marrow, unaccompanied by any increase in megakaryo- cytes. The necropsy findings in these patients are described and possible mechanisms for visceral fibrosis are discussed.

\section{Case report}

CASE 1

\section{Clinical course}

A $2470 \mathrm{~g}$ female infant with Down syndrome facies and hepatosplenomegaly was delivered by caesarean section because of fetal asphyxia at 38 weeks gestation. Peripheral blood examination at birth showed an increased white blood cell count $(128800 / \mu \mathrm{l})$ with $50 \%$ blasts. Other laboratory data showed high levels of lactate dehydrogenase $(7455 \mathrm{U} / 1)$ and slight liver dysfunction (aspartate aminotransferase $75 \mathrm{U} / 1$, alanine aminotransferase $106 \mathrm{U} / \mathrm{l}$ ).

Following conservative treatment with blood transfusion, total parenteral nutrition, diuretics, and antibiotics, the peripheral white blood cell count was decreased

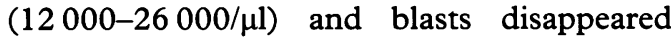
without antileukaemia treatment. The total bilirubin was $68.4 \mu \mathrm{mol} / 1$ on day 20 , and thereafter jaundice became severe (total bilirubin $657 \mu \mathrm{mol} / 1$, direct bilirubin $421 \mu \mathrm{mol} / 1$ on day 48). Serum aspartate and alanine aminotransferases were within the normal range from day 5 to day 20. The infant died of respiratory and liver failure on day 53 . There was no evidence of viral or syphilitic infection.

\section{Necropsy findings}

The liver showed diffuse intralobular and in-
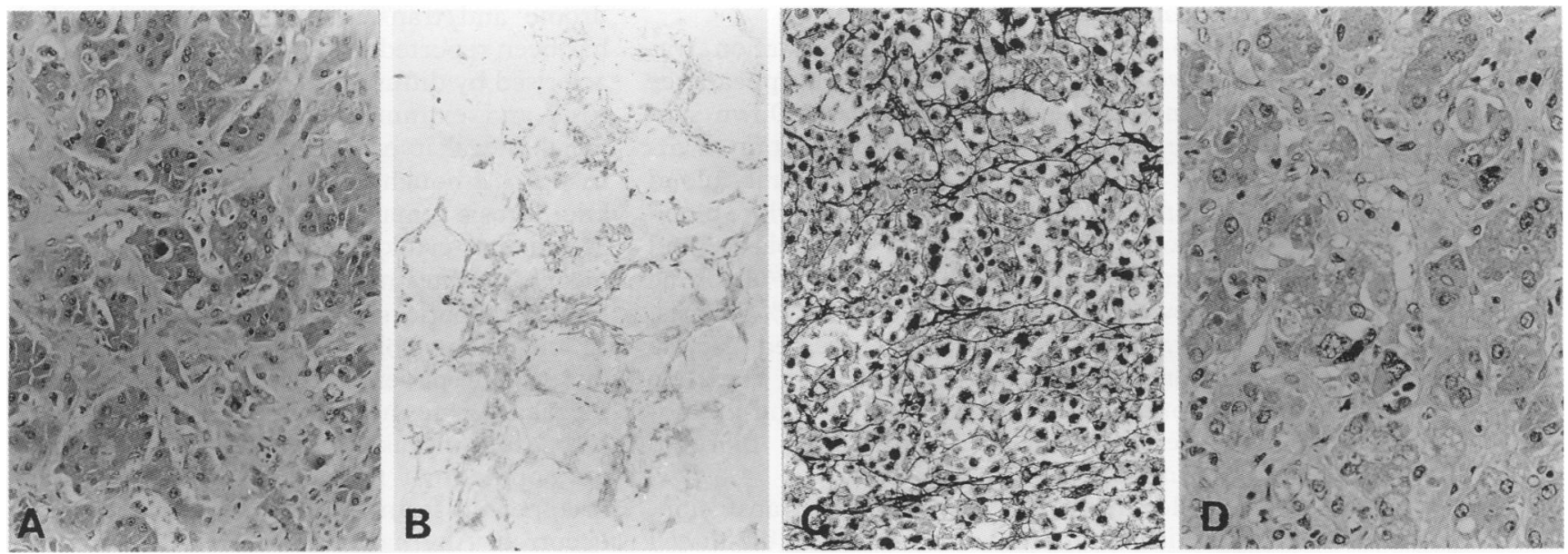

(A) Sinusoidal fibrosis surrounding small groups of hepatocytes and cholestasis (case 1, haematoxylin and eosin). (B) Immunostaining for $\alpha$-smooth muscle actin (case 1, streptavidin-biotin complex method). (C) Mild reticulin fibrosis of the bone marrow (case 1, silver impregnation). (D) Scattered atypical megakaryocytes in the fibrotic liver (case 2, haematoxylin and eosin). 
Case reports of infants with Down syndrome and transient abnormal myelopoiesis with hepatic fibrosis

\begin{tabular}{|c|c|c|c|c|c|c|c|c|}
\hline \multirow[b]{2}{*}{ Reference } & \multirow[b]{2}{*}{ Sex } & \multirow[b]{2}{*}{ Age at death } & \multicolumn{2}{|l|}{ Liver } & \multicolumn{3}{|c|}{ Bone marrow } & \multirow{2}{*}{$\begin{array}{l}\text { Pancreatic } \\
\text { fibrosis }\end{array}$} \\
\hline & & & Fibrosis & $M G K$ & Fibrosis & Cellularity & $M G K$ & \\
\hline Miller $^{2}$ & $F$ & 5 weeks & + & nd & nd & hyper & $\downarrow$ & + \\
\hline Nakagawa $^{3}$ & $\mathbf{F}$ & 77 days & + & nd & - & hypo & nd & nd \\
\hline \multirow[t]{2}{*}{ Becroft $^{4}$} & $F$ & 28 days & + & $+^{*}$ & - & normo & $\downarrow$ & + \\
\hline & M & & + & $+^{*}$ & - & normo & $\rightarrow$ & + \\
\hline \multirow[t]{4}{*}{ Miyauchi $^{1}$} & $\mathrm{~F}$ & 2 days & + & $+*$ & - & normo & $\rightarrow$ & nd \\
\hline & M & 10 days & + & $+^{*}$ & - & normo & $\rightarrow$ & nd \\
\hline & M & 41 days & + & $\pm^{* *}$ & - & normo & $\rightarrow$ & nd \\
\hline & $\mathrm{F}$ & 66 days & + & $+^{*}$ & - & normo & $\rightarrow$ & nd \\
\hline \multirow[t]{2}{*}{ Present cases } & $\mathrm{F}$ & 53 days & + & $\pm^{* *}$ & + & hyper & $\downarrow$ & + \\
\hline & $\mathbf{F}$ & 23 days & + & $+^{*}$ & + & hyper & $\rightarrow$ & + \\
\hline
\end{tabular}

$\mathrm{F}=$ female; $M=$ male; $M G K=$ megakaryocytic cells; $+^{*}=$ many; $\pm^{* *}=$ few; $\downarrow=$ decreased number; $\rightarrow=$ normal number; nd $=$ not described

terlobular fibrosis with no specific zonal distribution. The lobular hepatic architecture was not disturbed (figure, panel A). Elongated spindle cells with plump nuclei and collagen fibres were arranged along the hepatic sinusoids. These cells were immunopositive for $\alpha$-smooth muscle actin (figure, panel B; Dako) using the streptavidin-biotin complex technique. Intracellular and intracanalicular cholestasis was conspicuous and there were scattered foci of necrotic hepatocytes. Small numbers of mainly myeloid haematopoietic cells were located in the portal areas, but only very scanty megakaryocytes were found.

The bone marrow was hyperplastic, with increased numbers of myeloid cells in the main, some of which were immature in appearance. Megakaryocytes were decreased in number and normal in appearance, and there were no accumulations of blast cells. A slight diffuse increase in reticulin fibres was observed but showed no relationship to megakaryocytes (figure, panel C).

Extramedullary haematopoiesis consisting of all three haematopoietic cell lines was also found in the spleen, in which there was lymphoid depletion but no fibrosis. The pancreas showed mild perilobular and periacinar fibrosis but extramedullary haematopoiesis was not apparent. Both lungs showed bronchopneumonic changes in the upper lobes and massive haemorrhage in the lower lobes.

CASE 2

Clinical course

A $2434 \mathrm{~g}$ girl, born at 38 weeks gestation, was observed to have a mongoloid facial appearance and marked hepatosplenomegaly. Down syndrome was confirmed by demonstration of trisomy $21(47, X X,+21)$. Peripheral blood examination at birth showed a high white blood cell count $(89000 / \mu \mathrm{l})$ with $27 \%$ blasts, and an increased platelet count $(670000 / \mu \mathrm{l})$. Bone marrow aspiration showed $17 \cdot 5 \%$ blasts. Laboratory data showed increased lactate dehydrogenase (22924 U/l), aspartate aminotransferase (1135 U/l), alanine aminotransferase $(466 \mathrm{U} / \mathrm{l})$, and total bilirubin $(174 \mu \mathrm{mol} / \mathrm{l})$.

Following treatment with blood transfusion, diuretics, and antibiotics, the white blood cell count returned to normal and blasts disappeared on day 11. However, high values of lactate dehydrogenase $(3687 \mathrm{U} / \mathrm{l})$, aspartate aminotransferase (1079 U/l), alanine aminotransferase $(223 \mathrm{U} / 1)$ and total bilirubin ( $159 \mu \mathrm{mol} / \mathrm{l})$ still continued on day 22 . The patient died of gastrointestinal bleeding on day 23. There was no evidence of viral or syphilitic infection.

\section{Necropsy findings}

The liver showed diffuse sinusoidal and severe centrilobular fibrosis, but no evidence of cirrhosis. Small and large foci of necrotic hepatocytes were found in centrilobular areas. Intracellular cholestasis, fatty change, and tubular arrangements of hepatocytes were also observed. There was diffuse extramedullary haematopoiesis consisting of immature and mature myeloid cells and large numbers of atypical megakaryocytes (figure, panel D).

The bone marrow was hyperplastic with increased numbers of erythroid and myeloid series. Reticulin fibres were increased along bony trabeculae. Megakaryocytes were less marked and some were atypical, with large nonlobulated or multilobulated nuclei. No accumulation of blast cells was found. The spleen was congested and showed focal extramedullary haematopoiesis but no fibrotic changes. The pancreas showed mild interlobular and intralobular fibrosis accompanied by a few megakaryocytes.

\section{Discussion}

Hepatic fibrosis associated with Down syndrome and transient abnormal myelopoiesis has been reported previously (table). It is characterised by diffuse intralobular sinusoidal fibrosis and extramedullary haematopoiesis, in which megakaryocytes are prominent as in our case $2 .^{1}$ Although the pathogenesis of the hepatic fibrosis remains unclear, it has been suggested that cytokines produced by infiltrating megakaryocytes may play an important role. ${ }^{14}$ Similar hepatic sinusoidal fibrosis occurs in agnogenic myeloid metaplasia. ${ }^{5}$ However, in one of our patients (case 1) there were few megakaryocytes in the fibrotic liver. This could be because megakaryocytes associated with the development of fibrosis might have virtually disappeared from the liver by the time death occurred. Alternatively, factors other than the megakaryocytes might have been involved. One possible factor in the genesis of hepatic fibrosis is the treatment with total parenteral nutrition 
in this case. It is well known that histopathological changes in the liver damage of infants and children given total parenteral nutrition range from mild cholestasis to fibrosis or cirrhosis. ${ }^{6}$ Cholestasis may produce activation of Kupffer cells, stimulating proliferation and fibrogenesis of lipocytes which then show the structural characteristics of myofibroblasts, ${ }^{7}$ as found in our patients.

Proliferating blasts in transient abnormal myelopoiesis are known often to express antigens characteristic of megakaryocytes. ${ }^{8}$ Acute megakaryoblastic leukaemia (AMKL) is often accompanied by myelofibrosis with a marked increase in reticulin fibres. Although mechanisms of myelofibrosis are yet to be determined in AMKL, cytokines such as transforming growth factor $\beta,{ }^{9}$ as well as platelet derived growth factor, ${ }^{10}$ secreted from megakaryocytes can promote fibrogenesis. Both infants described here showed slight increases in reticulin fibre, a feature not described in previous reports of infants with Down syndrome, transient abnormal myelopoiesis, and hepatic fibrosis. ${ }^{1}$ This change might reflect an excessive release of cytokines or growth factors from proliferating haematopoietic cells, but not necessarily from megakaryocytes, since these were not increased.

1 Miyauchi J, Ito Y, Kawano T, Tsunematsu Y, Shimizu K Unusual liver fibrosis accompanying transient myeloproliferative disorder in Down's syndrome: a report of four autopsy cases and proposal of a hypothesis. Blood 1992;80:1521-7.

2 Miller JM, Sherrill JG, Hathaway WE. Thrombocythemia in myeloproliferative disorder of Down's syndrome. Pediatrics 1967;40:847-50.

3 Nakagawa T, Nishida H, Arai T, Yamada T, Fukuda $M$ Sakamoto S. Hyperviscosity syndrome with transient abSakamoto $S$. Hyperviscosity syndrome with transient abnormal my

4 Becroft DMO, Zwi LJ. Perinatal visceral fibrosis accompanying the megakaryoblastic leukemoid reaction of Down syndrome. Pediatr Pathol 1990;10:397-406.

5 Tsao M-S. Hepatic sinusoidal fibrosis in agnogenic myeloid metaplasia. Am f Clin Pathol 1989;91:302-5.

6 Cohen C, Olsen MM. Pediatric total parenteral nutrition liver pathology. Arch Pathol Lab Med 1981;105:152-6.

7 Friedman SL. The cellular basis of hepatic fibrosis: mech anism and treatment strategies. $N$ Engl $\mathcal{F}$ Med 1993;328: 1828-35.

8 Coulombel L, Derycke M, Villeval JL, Leonald C, BretonGorius J, Vial M. Characterization of the blast cell population in two neonates with Down's syndrome and transient myeloproliferative disorder. Br f Haematol 1987;66: 69-76.

9 Terui T, Niitsu Y, Mahara K, Fujisaki Y, Urushizaki Y, Mogi Y, et al. The production of transforming growth factor- $\beta$ in acute megakaryoblastic leukemia and its possible implications in myelofibrosis. Blood 1990;75: sible imp.

10 Castro-Malaspina H, Rabellino EM, Yen A, Nachman RL, Moore MAS. Human megakaryocyte stimulation of proliferation of bone marrow fibroblasts. Blood 1981;57 $781-7$.

\title{
Acute hepatic and renal failure caused by Pneumocystis carinii in patients with AIDS
}

\author{
R Boldorini, S Guzzetti, L Meroni, T Quirino, S Cristina, G Monga
}

\author{
Dipartimento \\ di Scienze Mediche, \\ II Facoltà di \\ Medicina e Chirurgia \\ di Novara-Università \\ di Torino, Italy \\ R Boldorini \\ S Cristina \\ G Monga \\ Istituto di \\ Scienze Biomediche \\ "L Sacco", \\ Università di Milano, \\ Milan, Italy \\ V Cattedra di \\ Anatomia Patologica \\ S Guzzetti \\ Clinica Malattie \\ Infettive \\ L Meroni \\ I Divisione \\ di Malattie Infettive \\ T Quirino \\ Correspondence to: \\ Dr Guido Monga, \\ Dipartimento di \\ Scienze Mediche-Via \\ Novara, Italy. \\ Accepted for publication \\ 13 March 1995
}

\begin{abstract}
Clinical and pathological findings are described in two AIDS patients with Pneumocystis carinii infection who received prophylactic treatment with nebulised pentamidine and developed unusual hepatic and renal failure. Histological examination showed clumps of $P$ carinii massively obstructing hepatic sinuses and portal vessels in the first patient, and merular and intertubular capillaries in the second. These findings could explain the unusual clinical features, characterised by acute hepatic and renal failure.

(f Clin Pathol 1995;48:975-978)
\end{abstract}

Keywords: AIDS, Pneumocystis carinii, hepatic failure, renal failure.

Disseminated pneumocystosis in patients with acquired immunodeficiency syndrome (AIDS) is reported with increasing frequency, ${ }^{1}$ but it appears still to be rare despite the high frequency of Pneumocystis carinii pneumonia. ${ }^{2} \mathrm{Ex}-$ trapulmonary localisation of pneumocystis has been reported in necropsy series and in single case reports, with the involvement of spleen, lymph nodes, bone marrow, liver, thyroid, gut, adrenal gland, skin, and brain. ${ }^{3}$

Extrapulmonary pneumocystosis can give rise to unusual clinical presentation, including thyroiditis, otitis media with mastoiditis, cutaneous lesions, "acute abdomen", hepatitis and choroiditis, and lymph node enlargement. ${ }^{4}$ To our knowledge, there are no well documented reports of renal failure due to $P$ carinii. Only a few cases of hepatic failure have been described on the basis of the clinical findings, ${ }^{5}$ but without morphological evidence of the type and severity of liver damage.

We describe the clinical and pathological features of two cases of disseminated pneumocystosis in AIDS patients who were given prophylactic treatment with nebulised pentamidine and developed hepatic and renal failure because of severe liver and kidney damage.

\section{Methods}

Necropsy examinations were performed 12 hours (patient 1) and 17 hours (patient 2) 